Published as:

Senge, M. O.; Davis, M. (2010):

Porphyrin (Porphine) - A neglected parent compound with potential.

Journal of Porphyrins and Phthalocyanines 14, 557-56

\title{
Porphyrin (Porphine) - A neglected parent compound with potential
}

\author{
Mathias O. Senge ${ }^{a, b^{*}}$ and Mia Davis ${ }^{a}$ \\ ${ }^{a}$ School of Chemistry, SFI Tetrapyrrole Laboratory, Trinity College Dublin, Dublin 2, Ireland \\ ${ }^{b}$ Medicinal Chemistry, Institute of Molecular Medicine, Trinity Centre for Health Sciences, Trinity College Dublin, St. \\ James's Hospital, Dublin 8, Ireland
}

Received date (to be automatically inserted after your manuscript is submitted)

Accepted date (to be automatically inserted after your manuscript is accepted)

Dedicated to Professor Karl M. Kadish on the occasion of his $65^{\text {th }}$ birthday

\begin{abstract}
Porphine is the parent compound of a family of biologically and chemically relevant compounds called porphyrins. The potential of these compounds is enormous and it would be advantageous to use the porphine (porphyrin) unit as a building block for the synthesis of diverse porphyrin complexes with a wide range of applications. However, despite first being synthesised over seventy years ago, porphine has not been utilized to its full extent due to low yield syntheses and poor solubility. Recent advances have now overcome many of these problems. The purpose of this review is to illustrate the advances made in porphine chemistry to illustrate the inherent potential of this simple compound.
\end{abstract}

KEYWORDS: Porphine, porphyrin, metalloporphyrin, tetrapyrroles. 


\section{INTRODUCTION}

Porphyrin chemistry is an ever expanding area of interest in the world of organic chemistry. Considerable progress has been made in synthesizing complex porphyrin molecules with a range of applications ranging from medicinal applications in photodynamic therapy to sensor and biosensors, to catalysis, to pigments and biomimetic models of enzymes. Porphyrins are found in nature and are involved in and play a major role in a wide variety of functions: oxygen transfer, electron transfer, oxidation catalysts and photosynthesis [1]. They are more well known for being essential components as hemes in hemoproteins in blood and chlorophylls in photosynthesis, both of which are involved in many biological processes including energy transfer and food production for the latter.

Although there have been many advances in the synthesis of substituted porphyrins and porphyrin oligomers in recent years, the synthesis of the parent porphyrin 1, otherwise known as porphine $\left(\mathrm{H}_{2} \mathrm{P}\right)$, still troubles organic chemists. Porphine is the simplest porphyrin and represents the core macrocycle of naturally occurring and synthetic porphyrins. Low yields and expense, however, make the synthesis of porphine on a large scale impractical. Another challenge synthetic chemist's faced was the high insolubility of porphine. The apolar tetrapyrrolic ring structure of porphine means it is poorly soluble in most organic solvents and hardly water soluble [2]. As a result of this almost all current chemical studies with porphyrins use the better soluble meso tetrasubstituted 2 or $\beta$-octasubstituted porphyrins

3.

However, new methods for synthesis of porphine have been developed recently and are under development [3]. This somewhat neglected compound should be an attractive target for chemists as its eight $\beta$-pyrrole and four meso positions, both with different reactivity profiles, offer the possibility to further elaborate porphyrins.

\section{SYNTHESIS OF PORPHINE}

\section{Early syntheses}

Historically, Thudichum [4] isolated the first porphyrin in 1867 by treating hemoglobin with sulfuric acid and this compound was later named by Hoppe-Seyler as hematoporphyrin. Fischer reported the first synthesis of porphyrin 1926, and subsequently prepared porphine in low yield by adding pyrrole- $\alpha$-aldehyde to boiling formic acid [5]. The spectroscopic results were confirmed by Paul Rothemund in 1936 [6]. He reported the synthesis of porphine upon addition of pyrrole in methanol to formaldehyde in methanol and pyridine under a nitrogen atmosphere. The yield he reported, although a breakthrough at the time, was still low and only $1 \mathrm{mg}$ of pure porphine could be recovered for every gram of pyrrole, giving a $0.1 \%$ yield. The desire to improve these yields has troubled chemists over the next 60 years.

In 1957, Steffan Krol improved the yield for the synthesis of porphine $\mathbf{1}$ with a novel one-step synthetic method in which he recovered $5 \%$ of the target material (Scheme 1). This was achieved by treating a dilute solution of 2hydroxymethylpyrrole 4 with potassium persulfate in glacial acetic acid [7]. The next significant step in the development of a porphine synthesis was taken by Longo and Thorne who reported the synthesis of $\mathbf{1}$ again via condensation of $\mathbf{4}$. They carried out kinetic studies and investigated solvent effects on the yield. They confirmed Alder and Beitchman's [8] proposal that the acid catalyzed condensation of $\mathbf{4}$ gave slightly higher yields than Krol's method and allowed for easier separation and isolation of porphine $\mathbf{1}$, as they received the highest yields when the reaction was carried out in an acidified aromatic solvent [9]. 
In 1997 a new synthetic route for the synthesis of porphine was developed by Ellis and Langdale from 4 which involves acidified water and immiscible organic solvent and oxidation was carried out using DDQ, resulting in yield of up to $13.6 \%$. It was noted that if the aqueous phase was eliminated, the yields of porphine dropped by up to $50 \%$ [10].

\section{One step syntheses}

$\beta$ substituted porphyrins are often synthesized by the MacDonald ' $2+2$ ' and ' $3+1$ ' approachs [11]. In the ' $3+1$ ' condensation, tripyranes, which are methylene bridged pyrrole trimers, represent the ' 3 ' component of the reaction and pyrrole aldehydes represent the ' 1 ' component. However, their synthesis can sometimes be troublesome and gives low yields due to the presence of two ester or carbonyl groups and unsubstituted tripyrrane proves even more troublesome. Here, a synthetic approach for porphine was inspired by Kämmerer [12] and his co-workers for the synthesis of calixarenes, a structure which resembles that of tripyrrane $\mathbf{6}$ and porphyrinogen.

Due to the high reactivity of 2,5-bis(hydroxymethyl)pyrrole 7 could only be isolated below room temperature in the presence of acid in water giving a yield of $61 \%$. The condensation of tripyrrane 6 with an equimolar amount of pyrrole according to the Lindsey method gave porphyrinogen in situ which was then oxidized to give $\mathbf{1}$ in good yield, relatively speaking of $31 \%$ [13].

One of most recent syntheses was reported by Lindsey and coworkers [14]. The reaction of 1-formyldipyrromethane 8 in the presence of air afforded (porphyrinato)magnesium(II) 9 in good yields of 30-40\% (Scheme 3). The advantages of this reaction include simplicity, high concentration, chromatography-free purification, gram scale synthesis and avoidance of the poorly soluble free base porphine. Due to the better solubility of the resulting magnesium complex it can now be used as a valuable core scaffold for derivatization and the free base porphine is readily obtained by demetallation using TFA and DCM.

Since the work of Dolphin it is well established that the chemical synthesis of porphyrins through condensation reactions proceeds first by formation of the porphyrinogen intermediate which is then oxidized to the aromatic compound [15]. This mechanism was questioned in 2001 by Sgamellotti et al who proposed an alternative mechanism based on theoretical calculations [16]. To date there is no experimental data to support this hypothesis.

\section{Muti-step syntheses/ Dealkylation}

5,10,15,20-Tetra(tert-butyl)porphyrin (10) [17,18] was first proposed as the precursor for the synthesis of 1 by Neya and Funasaki [19]. They proposed that de(tert-butyl)ation of the porphyrin would be possible using a proton and Lewis acid. This is because acid-labile tert-butyl groups can be used as protecting groups [20]. The classic example for such a reaction is the dealkylation of tert-butylbenzene to benzene [21]. A number of different acids were investigated in order to optimize the dealkylation of 10. The most effective catalyst was sulfuric acid in 1-butanol, as outlined in Scheme 4. A 1:1 mixture of the two gave the highest yield of $74 \%$ of unsubstituted porphyrin.

Dealkylation can also be carried out on tetra- $\beta$-(tert-butyl)porphyrin 11 again resulting in formation of 1 [22-24]. Compound 11 was first synthesized by Whitlock25 and serves as a precursor for the synthesis of porphine in the same fashion as porphyrin 10. Here the best method for dealkylation was sulfuric acid at a temperature of $190{ }^{\circ} \mathrm{C}$. Smooth dealkylation was observed after just $15 \mathrm{~min}$ (Scheme 4). The higher temperature requirement may be attributed to the $\beta$ -substituted molecule being a sterically less crowded macrocycle due to the tert-butyl groups being located on the less hindered $\beta$-pyrrole carbons. Meso substituted compounds such as $\mathbf{1 0}$ have highly ruffled macrocycles and easily 
undergo reactions at the meso position $[17,18,26]$. Thus, dealkylation of $\mathbf{1 0}$ is feasible at lower temperature due to its non-planarity. This rationale can be confirmed with thermogravimetric analyses [23,27]. Such dealkylations are just one example of electrophilic aromatic substitution of porphyrins which are discussed below.

Neya et al. followed up their elegant work by investigating the synthesis of porphine from 5,10,15,20tetrakis(hexyloxycarbonyl)porphyrin 12 [28]. This precursor porphyrin was synthesized from pyrrole and alkyl glyoxylate ester with yields $>6 \%$ under standard Lindsey conditions. Thermal decarboxylation of the hydrolyzed porphyrin proceeded smoothly. Ester hydrolysis and subsequent decarboxylation can be carried out in a one-pot procedure, using hot aqueous sulfuric acid (Scheme 4). A blue shift in the absorbance indicated the formation of porphine, which was then confirmed by further analytical studies. Yields for this method are comparable with or better than previous methods and the crystalline porphyrin was obtained in $77 \%$ yield.

It is worth noting that dealkylation is also an effective method for the synthesis of N-confused porphine [29]. Through a ' $3+1$ ' coupling of N-confused N-tert-butyl-tripyrrane and 7, N-protected NC-porphine was isolated. Removal of the N-tert-butyl group was carried out as described above for the dealkylation of $\beta$-tert-butylporphyrins with a reasonable yield of 55\%. N-confused porphine is planar and interestingly, the molecular structure, mode of packing and cell parameters are all very similar to that of porphine, in spite of the confused structure [29] Similarly to pophine, the solubility of $\mathrm{N}$-confused porphine is also low once it solidifies making it difficult to work with on a practical level.

\section{REACTIVITY}

\section{$\mathrm{S}_{\mathrm{E}} \mathrm{Ar}$}

Porphine readily undergoes electrophilic aromatic substitution and this is one of the most useful methods for functionalizing porphyrins. The first such investigation was reported in 1968 by Samuels et al. who studied the bromination, chlorination, and iodination of porphine [30]. It was found that direct chlorination of porphine resulted in low yields. However, reaction with bromine or $N$-bromosuccinimde (NBS) in chloroform yielded a mono-brominated compound and a $\beta$-substituted product with no liberation of free bromine. Bromo groups were also found to activate the opposite meso position of the porphine. The regioselectivity of these reactions can be explained in terms of FleischerWebb model for porphyrin aromaticity [31]. However, it was noted that if the brominating agent used was too strong, e.g., DBICA (dibromoisocyanurate) the yield of brominated product was very low. Using molecular bromine, no selectivity was observed. For bromination of 5-nitroporphyrin it was expected that the nitro group would direct the bromine group to the 10 position. This was not the case as the only compound isolated was the 5,15 -derivative; this is in line with the expectation that poorer selectivity is observed for stronger brominating agents [32].

Bromoporphyrins $(\mathbf{1 3 , 1 4})$ can deactivate the aromatic ring system inductively and activate the macrocycle by resonance. When the bromo group is in the 2 position of $\mathbf{1}(\mathbf{1 4})$, it causes an inductive deactivation of the ring. Porphine was found to be unaffected by iodine in chloroform and was destroyed by iodine-potassium iodate-sulphuric acid. Only use of iodine-silver sulphate-sulphuric acid resulted in the formation of monoiodoporphine. Halogenations of porphine are examples of true electrophilic substitution reactions with initial $\pi$-complex formation, collapse to a $\sigma$-complex followed by the loss of a proton to restore the aromaticity [33,34].

Nitration of porphine was first reported in 1974 by Drach and Longo [33]. It was found that by using stoichiometric amounts of nitric acid at $0{ }^{\circ} \mathrm{C}$, the mono-nitro derivative 15 was formed. The absence of the formation of $\beta$ - 
nitromaleimide confirmed that the porphine was not substituted in the $\beta$ position. If substitution had taken place in the $\beta$ position up to $25 \%$-nitro-maleimide would be formed due to degration of the macrocycle. The directing effect of the nitro group of the porphine was determined by nitrating mononitroporphine. A bathochromic shift was observed and further NMR analysis revealed that the sole compound isolated from this reaction was the meso disubstituted compound 16.

In 1973, Fuhrhop et al. carried out a study into the redox behavior of octaethylporphyrin (OEP) and used these data to predict and rationalize the reactivity of the porphyrin macrocycle [35]. It was deduced that porphyrin rings which have a relatively negative charge should undergo oxidation and other electrophilic substitution or addition reactions. Thus, it was concluded that an electropositive ring can be reduced and it should undergo nucleophilic additions and substitutions, e.g. photooxygenation of a porphyrin ring is only possible with the dianion, the magnesium or calcium complexes [36] Magnesium and zinc complexes are hydrolyzed easily under the conditions required for electrophilic substitution [35] The next most reactive metals towards electrophilic substitution are nickel and copper, in particular, they are very reactive towards Vilsmeier formylation which leads exclusively to the meso substituted product, e.g. compound 17 [37-40] Reagents such as $\mathrm{NO}_{2}^{+}, \mathrm{H}_{2} \mathrm{O}_{2}$ and $\mathrm{Br}_{2}$ are such strong oxidizing agents that very little metal specificity was observed, however, tin complexes were found to be the most stable in the presence of $\mathrm{Br}_{2}[41]$.

\section{$\mathrm{S}_{\mathrm{N}} \mathrm{Ar}$ reactions}

Nucleophilic substitution of porphyrins is a useful tool for the synthesis of various porphyrins [42]. Initially, such reactions were more limited to activated porphyrins. However, it was shown that use of organometallic reagents can be used to overcome these limitations [43] and this method proved to be a versatile entry into functionalized porphyrins [44-46]. The only exceptions are sterically hindered reagents and this method by now has allowed the preparation of numerous $\mathrm{A}_{\mathrm{x}}$ - and $\mathrm{ABCD}$-type porphyrins [47]. The reaction proceeds via initial reaction of the organic nucleophile with a meso carbon yielding an anionic species 19, which is then hydrolyzed to a porphodimethene or phlorin followed by oxidation with DDQ to yield the desired porphyrin 20 (Scheme 5) [48].

Follow up studies showed clearly that for unencumbered tetrapyrroles such as porphine prepared by the dealkylation method outlined above, both meso mono- [49] and 5,10-disubstituted porphyrins [50] are accessible by simple variation of the number of equivalents of the organometallic reagents used (Scheme 6) [51]. For example, for $n$-hexyllithium and $n \mathrm{BuLi}$ 1.2-1.5 equivalents were required for the mono-substitution of the porphine macrocycle. However, for the less reactive $\mathrm{PhLi}$ three equivalents was required and for the least reactive, 2-methoxyphenyllithium, 8 equivalents were required for mono-substitution. Note that improved yields are obtained with nickel(II) complexes [51].

Potentially, further synthetic applications may arise from this reaction through trapping of the intermediary anion with electrophiles, [52] transformation into meso-meso-linked bisporphyrins, [53] utilization of thermodynamically controlled reactions, [54] or $\beta$-addition of sterically hindered reagents [55] to yield porphine derivatives akin to those observed for meso-substituted porphyrins [42].

\section{REDOX CHEMISTRY}

Redox reactions catalyzed by porphyrins are of great interest in a variety of fields, ranging from biology and photosynthesis to electrocatalysis [56-60]. Redox reactions are relevant in the biochemistry of heme proteins and in 
artificial catalytic systems. One of the striking features of metalloporphyrins in general is their ability to undergo facile reduction and oxidation. Numerous studies have shown that small structural differences in metalloporphyrins allow fine tuning of their activity and selectivity. Using various transition metals in different oxidation states as the central atom of porphine could modify the catalytic performance of metalloporphines [61]. The basic redox chemistry of porphyrin and related compounds has been reviewed before $[62,63]$.

One early investigation into the gas-phase synthesis of metalloporphine ions found that many metal ions, both bare and ligated, react with porphine vapor to produce the corresponding metalloporphine cations and anions in good yield [64] $\mathrm{Fe}^{+}$was generated by excimer laser $(380 \mathrm{~nm})$ ablation of an iron disk and reacted with porphine to generate Fe $(\mathrm{P})^{+}$ and hydrogen. Exothermic charge transfer from $\mathrm{Fe}^{+}$to porphine was found to generate $\mathrm{H}_{2} \mathrm{P}^{+}$also. For metalloporphine anions a different approach was required because no atomic anions are generally obtained from laser ablation of metals. Dissociative electron attachment to metal compounds often yields reactive ligated anions, e.g., electron attachment to porphine yielding $\mathrm{Ni}(\mathrm{P})^{-}$. Further attempts were made to oxidize $\mathrm{Fe}(\mathrm{P})^{+}$to produce $\mathrm{Fe}(\mathrm{P}) \mathrm{O}^{+}$as a gas phase model for reactive centers of enzymes, but were unsuccessful. The closest they got to the desired compound which could be isolated was $\mathrm{Fe}(\mathrm{P}) \mathrm{OH}^{+}$which was formed as a minor product in the slow reaction between $\mathrm{Fe}(\mathrm{P})^{+}$and tert-butyl hydroperoxide.

In nature, oxygen reduction takes place at soft interfaces such as biomembranes that provide both a physical separation of the reactants and products, and an electrochemical driving force resulting from the membrane electrical potential difference. The polarization of the soft interface also allows an electrochemical control for different charge transfer reactions such as ion transfer, assisted ion transfer and heterogeneous electron transfer reactions between a hydrophilic and lipophilic redox couple. In classic molecular electrocatalysis, porphyrin catalysts are usually deposited on solid electrodes and their catalytic activity is observed as a shift of the reduction potential to higher electrode potential values. One example is cobalt porphine $(\mathrm{CoP})$ which has been shown to catalyze a four electron reduction of $\mathrm{O}_{2}$ and $\mathrm{H}_{2} \mathrm{O}$ when being adsorbed on graphite electrodes, and to catalyze mainly a two-electron reduction of $\mathrm{O}_{2}$ to yield hydrogen peroxide when being deposited onto gold electrodes. Both of these processes are proton-coupled electron transfer reactions, where proton transfer and electron transfer are tightly coupled [58]

Another publication showed that the redox properties of metalloporphines can be modulated by ring substitution. For example, if porphine is substituted with $-\mathrm{NO}_{2}$ an increase in the energy needed to oxidize the metalloporphyrin was observed. The opposite was the case for $-\mathrm{Br}$ and $-\mathrm{OCH}_{3}$ substitution. Here the electron acceptor substitution produces a metal centre less prone to lose electrons, stabilizing the reduced form, while substitution of porphine with an electron donor produces metalloporphyrins with a more stable oxidized form [59] Clearly, strong modification in the redox properties of metalloporphyrins can be achieved by substituting the macrocycle with appropriate groups. For Fe porphine a higher binding capability was observed than for substituted porphyrins. It was suggested that this was due to back donation effects. These results were confirmed in theoretical studies on iron and manganese porphines. Here, it was noted that the change of the oxidation state of the central atom can influence the geometry and electronic structure of the molecule, and particularly its oxygen-binding properties [64].

\section{STRUCTURAL CHEMISTRY}

The first crystal structure of porphine was reported by Webb and Fleischer $[31,65]$. The molecular structure in the crystal was characterized by an almost planar macrocycle and the $C_{\alpha}-C_{b}$ bonds were found to be equal. Although the 
structure was disordered, intermolecular differences in pyrrole rings were found to be small. There was no evidence that the observed planarity of porphine was apparent and achieved by random distribution of nonplanar molecules in the crystal.

Upon examination, a parallel overlapping of the molecule at the graphite layer separation was seen in pairs. Although similar to that of phthalocyanine, at that time, the packing for porphine was considered relatively rare for compounds of the same dimensions. Hence despite being so closely packed, porphine exhibits no close inter-molecular contact. Initially, the effects of any one of the three factors which influence the equilibrium configuration of the molecule could not be examined alone in an experimental situation; the $\sigma$ bonding, $\pi$ bonding and packing forces. It was also speculated that the vibrational force normal to the plane may cause deviations from planarity but ruffling can be attributed to crystal-packing forces in addition to the $\sigma$ bonding effects.

A number of theories were proposed regarding the planarity of the macrocycle as a result of this study. The first of these is that a ruffled configuration of the porphine skeleton in the 'free unconstrained' porphine macrocycle is stable at low temperatures. It was proposed that although the porphine macrocycle may appear planar due to the packing forces, ruffling is in fact favored by the presence of $\sigma$-bonding in porphine as well as the $\pi$ bonding. This relationship between $\sigma$ bonding and ruffling is a result of the angular strain in the $\sigma$ bonds that can not be minimised in the planar porphine conformation. These angular strains can also contribute to the deformation of the porphine skeleton enabling porphine to be adaptable in a variety of biological environments. Another theory stated that the porphine skeleton in the free unconstrained molecule is planar and deviations from planarity are caused by crystal-packing forces, i.e. close contact with neighboring molecules.

The next study on the crystal structure of porphine was carried out by Chen and Tulinsky in 1971 [66]. The molecule was determined to have a planar macrocycle and it was concluded that the previous structural report discussed above had discrepancies. It was suggested that the inclusion of a metal impurity into the structure factor calculations resulted in a decrease of the conventional $\mathrm{R}$ factor of about 0.09 but concluded that their work was approximately correct. Later Gross et al. reported another structure of a crystal obtained during an attempted synthesis of corrole [67] They found that the inner hydrogen atoms were localized on two particular pyrrole nitrogen atoms at room temperature, as opposed to disordered location on all four nitrogen atoms in the earlier studies. The crystal structure is characterized by the formation of overlapping pairs, which are arranged in a herringbone manner in three-dimensional space; clearly the result of intermolecular $\pi$ - $\pi$ interactions (Fig. 1).

Scheidt and coworkers reported the structure of (porphyrinato)nickel(II) which turned out to be quite surprising [68] Typically for substituted Ni porphyrins a ruffled nonplanar conformation is expected as a result of the Ni-N bond shortening [69-71]. Here, the crystal structure of the parent nickel(II) porphyrin exhibited a planar porphyrin macrocycle with a $\pi-\pi$ packing arrangement and a small lateral shift [68]. The average deviation of the macrocycle atoms from their least-squares-plane $(\Delta 24)$ was only $0.02 \AA$ and only very minor ruffling contributions were observed. Resonance Raman spectroscopy confirmed that the planar conformation is preferred both in the solid state and in solution.

Other reported crystal structures include an essentially planar (dimethoxy)(porphinato)germanium(IV) [72] and a highly disordered dimeric free base porphyrin assembly in coordination cages [73]

Recent studies have shown that the aromaticity of the two protonated pyrrole rings which is higher than that of the pyrrole rings without an inner hydrogen $[74,75]$. Deprotonation of $\mathrm{H}_{2} \mathrm{P}$ leads to the dianion $\mathrm{P}^{2-}$, in which the charge is distributed over the whole molecule in $\mathrm{D}_{4}$ symmetry. The aromaticity of the pyrrolic rings is reduced upon removal of the NH hydrogen atoms; however, the aromaticity of the inner ring remains the same (Figure 2). For both $\mathrm{P}^{2-}$ and $\mathrm{H}_{2} \mathrm{P}$, Hückel's rule for aromaticity $(4 n+2)$ is obeyed with 18 contributing $\pi$ electrons for both species. To obtain the neutral deprotonated porphine (Por), the removal of two electrons causes localization of the $\pi$ electrons resulting in a change of 
symmetry to $\mathrm{D}_{2 \mathrm{~h}}$, thus, Por cannot be considered an aromatic species. Por is consistent with the (4n) rule of antiaromaticity and $\mathrm{P}^{2-}$ obeys rules of aromaticity [76]. The diatropic ring current of $\mathrm{P}^{2-}$ and paratropic ring current of Por was reported by Steiner and co workers [77].

A density functional theory study on the chemical bonding and aromaticity of metalloporphines using first row (Sc $\mathrm{Zn})$ and second row (Ru, Pd, Ag, Cd) transition metals as well as alkaline-earth metals (Mg, Ca) was reported [76] For all cases the orbitals and densities were initially described by $\mathrm{C}_{2 \mathrm{v}}$ symmetry, which ended up as $\mathrm{D}_{4}$ symmetry after optimisation. For $\mathrm{Ca}, \mathrm{Cr}, \mathrm{Ni}$, and $\mathrm{Sc}$ the most stable configuration was found with the metal ion displaced from the porphine plane $\left(\mathrm{C}_{4}\right.$ symmetry). For $\mathrm{Cr}$ and $\mathrm{Ni}$, this displacement distortion has no effect because it occurs at the triplet state which is less stable than the corresponding ground state. The ground spin state of transition metal complexes can be explained in terms of Hund's rule of maximum multiplicity and metal-ligand interactions. Explorative data analysis (EDA) has been used to study the metal-ligand interactions in terms of the heterolytic association reaction between the cationic metals and the anionic porphine ligand. It was confirmed that the interaction between the metal and porphine is electrostatic in nature. Pauli repulsion interactions were also observed in all cases except for MgP. In most cases, the orbital interactions are large enough to overcome the Pauli repulsion, thus contributing in a positive nature to the M-P interactions. The exceptions to this are $\mathrm{CaP}$ and $\mathrm{MgP}$ for which the covalent orbital interactions are much reduced. For $\mathrm{CaP}$, the metal ligand interaction is purely electrostatic, which is not the case for $\mathrm{MgP}$ as the small covalent orbital interactions are substantially larger than Pauli repulsion. It was concluded from these findings that for all MP's (except $\mathrm{CaP}$ ), the covalent interactions are one of the most important factors regarding the stability of the complex. The fact that $\mathrm{CaP}$ only possess electrostatic interactions may be an explanation as to this is the only complex in which the metal is found so far away from the porphine plane with the longest M-N bond lengths [76].

As previously discussed the outer ring of $\mathrm{P}^{2-}$ is less aromatic due to the localization of the electrons in the $\mathrm{C}-\mathrm{C}$ bonds. However, aromaticity increases significantly upon metal complexation due to polarization of the $\pi$ cloud and charge transfer. The polarization of the $\pi$ cloud is caused by the divalent positive charge of the metal that moves the more polarisable $\pi$-electrons near to the $\mathrm{N}$ atoms. The aromaticity of the complexes is actually quite close to that of $\mathrm{H}_{2} \mathrm{P}$.

\section{SPECTROSCOPIC AND PHYSICAL PROPERTIES}

The spectra of all porphine complexes are dominated by the $\pi-\pi^{*}$ transitions. There is a relatively weak band (Q) in the visible, often with broad vibrational structure, and an intense Soret band in the near UV. These bands have most successfully described by Gouterman's four-orbital model [78] that mixes configurations generated from $a_{1 u}(\pi) \rightarrow$ $e_{\mathrm{g}}\left(\pi^{*}\right)$ and $\mathrm{a}_{2 \mathrm{u}}(\pi) \rightarrow e_{\mathrm{g}}\left(\pi^{*}\right)$ frontier orbitals. These two excited configurations are of $\mathrm{E}_{u}$ symmetry and are predicted to have nearly equal intensity. However, they will strongly interact resulting in a weaker Q band and strong Soret band. The two triplets from these two excitations do not mix directly with each other and are believed to lie below the Q band energy. There are two other triplets which are generated from the excitations out of the orbitals $b_{2 u}(\pi) a_{1} a_{2 u}(\pi)$ symmetry into the LUMO $e_{\mathrm{g}}\left(\pi^{*}\right)$. The higher of these triplets was calculated to be nearly degenerate with the Soret band energy and detailed calculations and comparisons with the various theories have been reported for Fe(II) porphyrins [79]. 
Calculations of the vibrational frequencies and normal modes in the low lying iron spin states of (porphyrinato) iron showed that there are only five modes carrying large amplitude motions of the iron atom. Three of these are out-ofplane modes of $\mathrm{a} 2_{\mathrm{u}}$ symmetry, of which the mode of the lowest frequency $\left(\gamma_{9}\right)$ is the doming motion of the complex. The calculated frequency was close to that observed in coherent reaction dynamics in heme proteins. The frequency of this motion was lower in high-spin than in low- or intermediate spin states of the complex. Identification of modes with large-amplitude motion of the iron atom as low-frequency out-of-plane $a_{2 u}$ indicated that the iron atom motion was mostly one-dimensional, acting as a switch between planar and domed structures. Calculations have confirmed the existence of a crossing between states of intermediate- and high-spin multiplicity at domed configurations of the complex [80]

Nickel(II) porphyrins have been investigated in more detail and an X-ray absorption and photoemission study on a comparison of $\mathrm{Ni}(\mathrm{II})$ porphine and $\mathrm{Ni}(\mathrm{II}) \mathrm{N}$-confused porphine was reported [81]. The former has $\mathrm{D}_{4 \mathrm{~h}}$ symmetry and possesses four $\pi$ bonds due to in-plane mixing of the ligand $2 \mathrm{p} \sigma$ states with $\mathrm{Ni}_{3} 3 \mathrm{~d}_{\mathrm{z} 2}+4 \mathrm{~s}, 3 \mathrm{~d}_{\mathrm{x} 2-\mathrm{y} 2}$ and $4 \mathrm{p}_{\mathrm{x}, \mathrm{y}}$ states which are described by the $a_{1 g}, b_{1 g}$ and $e_{u}$ molecular orbitals. In this case the $a_{1 g}$ and $b_{1 g}$ represent the HOMO and LUMO, respectively. The $\pi$-molecular orbital of $\mathrm{e}_{\mathrm{g}}$ symmetry represents the covalent bond between the metal atom with the ligands which is accompanied by a charge transfer from the metal atom into the ligands ( $\pi$-back-donation). The difference between the absorption spectra of Ni metal and NiP were explained within the framework of a quasimolecular approach with inclusion of covalent mixing between the two valence electron states. A high energy absorption shift in going from the Ni metal to NiP is a direct result of the decrease in the effective number of $3 \mathrm{~d}$ electrons on the $\mathrm{Ni}$ atom due to the strong $\mathrm{Ni} 3 \mathrm{~d}$-ligand $2 \mathrm{p} \pi$-type covalent bonding. This causes delocalization of the $3 \mathrm{~d}$ states, thus decreasing the electron density on the Ni atom as well as screening of the Ni $2 \mathrm{p}-3 \mathrm{~d}$ electron transitions. The metal-to-ligand charge transfer in NiP occurs from the occupied $\mathrm{Ni} 3 \mathrm{~d}_{\mathrm{xz}, y z} e \pi$ to unoccupied ligand $2 \mathrm{p} \pi^{*}$ orbitals resulting in the corresponding absorption band.

Raman spectroscopy has also become a useful spectroscopic tool for the analysis of porphine. One such example is a study of Ni (II) porphine adsorbed on aqueous silver sol [82]. For NiP, its two lowest excited states (Soret and Q) that are both doubly degenerate with $e_{u}$ symmetry. The electronic transitions from the ground state to the excited states are $\pi-\pi^{*}$ and are polarized within the porphine plane. The Frank-Condon mechanism dictates the enhancement of totally symmetric $\mathrm{a}_{1 \mathrm{~g}}$ modes when excitation is tuned into the strongly allowed Soret absorption. Vibronic coupling within one $e_{\mathrm{u}}$ excited state, either Soret or Q contributes to the enhancement of $\mathrm{a}_{1 \mathrm{~g}}, \mathrm{~b}_{1 \mathrm{~g}}$ and $\mathrm{b}_{2 \mathrm{~g}}$ modes (Jahn-Teller effect). However, vibronic coupling between Soret and Q states contributes to the enhancement of modes with $a_{1 g}$, $a_{2 g}$, $b_{1 g}$ and $\mathrm{b}_{2 \mathrm{~g}}$ symmetry (Herzberg-Teller effect).

Detailed fluorescence and optical studies on various metallo porphines were reported by Bohandy and coworkers in the late seventies [83-85]. Further investigations of the phosphorescence on PdP in a matrix were carried out by A. Starukhin and co-workers [86,87]. They traced two PdP macrocycle conformations in the fine structure phosphorescence spectra in a large number of Shpol'skii matrices at liquid helium temperature. It was shown that the short-wavelength (planar conformation) and long-wavelength (distorted conformation) could be stabilized by choosing the appropriate matrix. Thus it was concluded that the matrix can influence the conformation of the macrocycle [88].

\section{OUTLOOK AND APPLICATIONS}


Porphyrins have many applications some of which include chemistry, medicine and biomimetic synthesis. In chemistry, the applications include light conversion, autoxidation catalysis, sensors, nonlinear optical materials and light emitting diodes. In medicine, they can be used for cytochrome oxidase models, phototoxicity, tumor therapy and virus eradication. In principle, porphines can be used in all of these areas and their improved synthesis has opened the doors for the potential synthesis of more complex systems with porphine building blocks. Theoretical studies have targeted the interaction of porphine and its metal complexes with fullerenes. C60 covalently or noncovalently bound to porphine may be used in light harvesting photosynthetic systems, data storage media, photovoltaic and electrochemical devices, and gas sensors [89]. Likewise the nanoworld has discovered porphine and metalloporphines, a first study investigated their surface structures. For Ni(II) porphine a well-ordered molecular layer in which the porphine molecules have a flat orientation with the molecular plane lying parallel to the substrate and forming a hexagonal overlayer on the surface was observed [90]. A related study using $\operatorname{Ag}(111)$ surfaces at room temperature showed that

$\mathrm{Ni}(\mathrm{II})$ porphine grows defined nanolines on the surface indicating the potential of using these systems for nanostructured materials [91]. The true potential of porphines and their metal complexes is just emerging and hopefully the advances made in their synthesis will now spawn advances in their characterization and applications.

\section{ACKNOWLEDGEMENTS}

Writing of this article and our own work described therein was generously supported by grants from SFI; most recently by a Science Foundation Ireland Research P.I. (SFI 09/IN.1/B2650) grant.

\section{REFERENCES}

1. Kadish KM, Smith KM and Guilard R. The Porphyrin Handbook; Academic Press: Boston, 2000; Vol. 1-10.

2. $\quad$ Berlein HP and Müller GJ. Applied Laser Medicine; 2003: 275-276

3. Senge MO and Richter J. J. Porphyrins Phthalocyanines 2004; 8: 934-953; Horn S, Dahms K, Senge MO.; J. Porphyrins and Phthalocyanines 2008; 12; 1053-1077.

4. Thudichum JLW. Rpt. Med. Off. Privacy Council, 1867, App. 7, 10, 152.

5. Fischer H and Gleim W. Liebigs Ann. 1936; 521: 157-160.

6. Rothemund P. J. Am. Chem. Soc. 1936; 58, 625-627.

7. Krol S. J. Org. Chem 1959; 24: 2065-2067.

8. Beitchman SD; PhD Thesis, University of Pennsylvania, 1966.

9. Longo FR and Thorne EJ. J. Heterocyclic Chemistry 1975; 12: 1305-1309.

10. Ellis Jr PE and Langdale WA. J. Porphyrins Phthalocyanine 1997; 1: 305-307.

11. Lash TD. Chem. Eur. J. 1996; 2: 1197-1200.

12. Happel G, Mathiasch B and Kämmerer H.; Makromol. Chem. 1975; 176: 3317-3334; Böhmer V, Chhim P and Kämmerer H. Makromol. Chem. 1979; 180: 2503-2506.

13. Taniguchi S., Hasegawa H ., Nishimura M., Takahashi M. Synlett 1999: 73-74.

14. Lindesey JS, Dogutan DK and Ptaszek M. J. Org. Chem. 2007; 72: 5008-5011.

15. Wijesekera TP, Paine JB, Dolphin D, Einstein FWB, Jones T J. Am. Chem. Soc; 1983: 105: 6747-6749; Mashiko T. and Dolphin D. Comprehensive Coordination Chemistry, eds. Wilkinson G., Gillard RD. and McCleverty JA, Pergamon, Oxford, 1987; 2: 21.1: 855. 
16. Sgamellotti A, Rosi M, Belanzoni, P, Bonomo L and Floriani C. J. Chem. Soc., Dalton Trans. 2001: 9; 14921497.

17. Ema T, Senge MO, Nelson NY, Ogoshi H and Smith KM. Angew. Chem., Int. Ed. Engl. 1994; 33: $1879-1881$.

18. Senge MO, Bischoff I, Nelson NY and Smith KM. J. Porphyrins Phthalocyanines 1999; 3: 99-116.

19. Neya, S; Funasaki, N. Tetrahedron Lett. 2002; 43: 1057-1058.

20. Saleh SA and Tashtoush HL. Tetrahedron 1998; 54: 14157-14177.

21. Roberts RM, Baylis EK and Fonken GJ. J. Am. Chem. Soc. 1963; 85: 3454-3458.

22. Neya S, Quan JS, Hoshino T, Hata M and Funasaki N. Tetrahedron Lett. 2004; 45: 8629-8630.

23. Hatscher SS. Ph.D. Dissertation, Freie Universität Berlin, Germany, 2003.

24. Senge MO, Ryppa C, Hatscher S, Kleinpeter E, Wacker P, Schilde U and Wiehe A. Chem. Eur. J. 2005; 11: 3427-3442.

25. Whitlock BJ, Whitlock HW and Alles H. J. Am. Chem. Soc. 1974; 96: 3959-3965.

26. Senge MO, Ema T and Smith KM. J. Chem. Soc., Chem. Commun. 1995: 733-734.

27. Kuś P and Stefaniak M. Monatsh. Chem. 2004; 135: 509-511.

28. Neya S, Quan JS, Hata M, Hoshino T and Funasaki N. Tetrahedron Lett. 2006; 47: 8731-8732.

29. Morimoto T, Taniguichi S, Osuka A and Furuta H. Eur. J. Org. Chem. 2005; 3887-3890.

30. Samuels E, Shuttleworth R and Stevens TS. J. Chem. Soc. 1968: 2:145-147.

31. Webb LE and Fleischer EB. J. Am. Chem. Soc. 1965; 87: 667-669.

32. Longo F, Nudy LR and Schieber C. Heterocycles 1987; 26: 1797-1803.

33. Longo F and Drach JE. J. Org. Chem.1974; 39: 3282-3284.

34. Longo F, Nudy LR and Coffey JC. J. Heterocycl. Chem.1982; 19: 1589-1590.

35. Furhop J-H, Kadish KM and Davis DG. J. Am. Chem. Soc. 1973; 95: 5140-5147.

36. Wasser, PKW and Fuhrhop J-H. Ann. N. Y. Acad. Sci. 1973; 206: 533-548.

37. Inhoffen HH, Fuhrhop J-H, Voigt H and Brockmann Jr H. Liebigs Ann. Chem. 1966; 695: 133-143.

38. Johnson AW and Oldfield D. J. Chem. Soc. C 1966: 794-798.

39. Dahms K, Senge MO and Bakar MB. Eur. J. Org. Chem. 2007: 3833-3848.

40. Schlözer R and Fuhrhop J-H. Angew. Chem., Int. Ed. Engl. 1975; 14: 363.

41. Fuhrhop J-H and Lumbantobing T. Tetrahedron Lett. 1970: 32: 2815-2818.

42. Senge MO. Acc. Chem. Res. 2005; 38: 733-743.

43. Senge MO and Kalisch WW. Angew. Chem., Int. Ed. Engl. 1998; 37: 1107-1109.

44. Senge MO and Feng XD. J. Chem. Soc., Perkin Trans. 1 2000: 3615-3621.

45. Senge MO, Kalisch WW and Bischoff I. Chem. Eur. J. 2000; 6: 2721-2738.

46. Feng X and Senge MO. J. Chem. Soc., Perkins Trans. 1 2001: 1030-1038.

47. Senge MO, Shaker YM, Pintea M, Ryppa C, Hatscher SS, Ryan A and Sergeeva Y. Eur. J. Org. Chem. 2010: 237-258.

48. Feng X, Bischoff I and Senge MO. J. Org. Chem. 2001; 66: 8693-8700.

49. Wiehe A, Ryppa C and Senge MO. Org. Lett. 2002; 4: 3807-3809.

50. Hatscher S and Senge MO. Tetrahedron Lett. 2003, 44: 157-160.

51. Senge MO, Ryppa C, Hatscher S, Kleinpeter E, Wacker P, Schilde U and Wiehe A. Chem. Eur.J. 2005 ; 11: $3427-$ 3442.

52. Feng X and Senge MO. Tetrahedron 2000; 56: 587-590.

53. Senge MO and Feng X. Tetrahedron Lett. 1999; 40: 4165-4168. 
54. Bischoff I, Feng X and Senge MO. Tetrahedron 2001; 57: 5573-5583.

55. Sergeeva, NN and Senge MO. Tetrahedron Lett. 2006; 47: 6169-6172.

56. Bottomley LA, Olson L and Kadish KM. Adv. Chem. Ser. 1982; 201: 279-311.

57. Saveant JM. Chem. Rev. 2008; 108: 2348-2378.

58. Hatay I, Su B, Li F, Méndez MA, Khoury T, Gros CP, Barbe JM, Ersoz M, Samec Z and Girault HH. J. Am. Chem. Soc. 2009; 131: 13453-13459.

59. Estrin DA, Bikiel DE, Bari SE, Doctorovich F and Estrin DA. J. Inorg. Biochem. 2008; 102: 70-76.

60. Liao MS and Scheiner S. J. Comput. Chem. 2002; 23: 1391-1403.

61. Rutkowska-Zbik D and Witko M. J. Mol. Catal. A 2006; 258: 376-380.

62. Fuhrhop JH Structure and Bonding: 1974; 18: 1-67

63. Kadish, K. M. The Porphyrin Handbook: 2000; 3; 405-420.

64. Beauchamp JL and Irikura KK. J. Am. Chem. Soc.;1991; 113: 2767-2768.

65. Fleischer EB and Webb LE. J. Chem. Phys. 1965; 43: 3100-3111.

66. Chen BML and Tulinsky A. J. Am. Chem. Soc. 1972; 94: 4145-4151.

67. Saltsman I, Goldberg I, Balasz and Gross Z. Tetrahedron Lett. 2007; 48: 239-244.

68. Jentzen W, Turowska-Tyrk I, Scheidt WR and Shelnut JA. Inorg. Chem. 1996; 35: 3559-3567.

69. Hoard, JL. Ann. N.Y. Acad. Sci. 1973; 206: 18-31.

70. Lee YJ and Scheidt WR. Struct. Bonding (Berlin) 1987; 64: 1-70.

71. Senge MO, Renner MW, Kalisch WW and Fajer J. J. Chem. Soc., Dalton Trans. 2000: 381-385.

72. Mavridis A and Tulinsky A. Inorg. Chem. 1976; 15: 2723-2727.

73. Ono K, Yoshizawa M, Kato T, Watanabe K and Fujita M. Angew. Chem. Int. Ed. 2007; 46: 1803-1806.

74. Cyrański MK, Krygowski TM, Wisiorowski M, Hommes NJRV and Schleyer PvR. Angew. Chem., Int. Ed. 1998; 37: $177-180$.

75. Campomanes P, Menéndez MI, Cárdenas-Jirón GI and Sordo TL. Phys. Chem. Chem. Phys. 2007; 9: 5644-5648.

76. Feixas F, Solà M and Swart M. Can J. Chem. 2009; 87: 1063-1073.

77. Steiner E and Fowler PW. ChemPhysChem 2002; 3: 114-116.

78. Gouterman M. J. Mol. Spectrosc. 1961; 6: 138-163.

79. Zerner MC, Edwards WD and Weiner B. J. Am. Chem. Soc. 1986; 108: 2196-2204.

80. Spiro TG, Kozlowski PM, Berces A and Zgierski MZ. J. Phys. Chem. 1998; 102: 2603-2608.

81. Kranikov SA, Sergeeva NN, Brzhezinskaya MM, Preobrajenski AB, Sergeeva YN, Vinogradov NA, Cafolla AA, Senge MO and Vinogradov AS. J. Phys. Cond. Matter 2008; 20: 235207.

82. Li X., Petrov VI., Chen D., Yu N. J. Raman Spectrosc. 2001; 31: 503-519.

83. Bohandy J and Kim BF. J. Chem. Phys. 1980; 73, (11) 5477-5481.

84. Bohandy J. and Kim BF. J. Chem. Phys. 1982; 76: 1180-1181.

85. Kim BF. and Bohandy J. J. Mol. Spectrosc. 1978; 73: 332-343.

86. Starukhin A, Kruk M and Cerwieniec R. J. Luminesc. 2008, 128: 531-536.

87. Starukhin AS and Kruk NN. Opt. Spectrosc. 2007, 103: 317-321.

88. Starukhin A, Kruk, M and Czerwieniec R. Proc. SPIE 2007; 6727: 67272.

89. Basiuk VA. J. Phys. Chem. A 2005; 109: 3704-3710.

90. Beggan JP, Krasnikov SA, Sergeeva NN, Senge MO and Cafolla AA. J. Phys. Cond. Matter 2008; 20: 015003.

91. Krasnikov SA, Beggan JP, Sergeeva NN, Senge MO and Cafolla AA. Nanotechn. 2009; 20: 135301. 


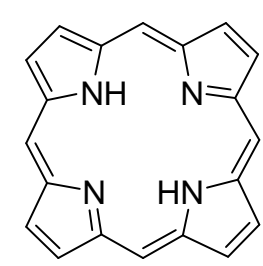

1

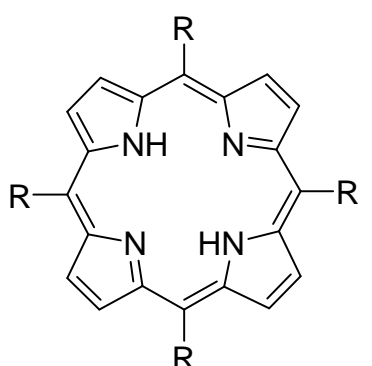

2

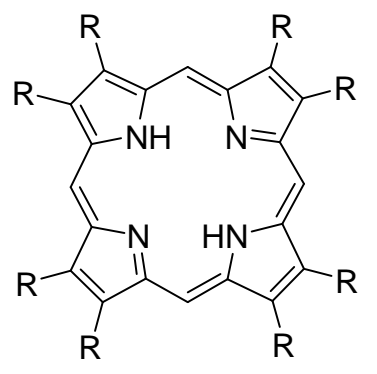

3

Formula 1

4

4

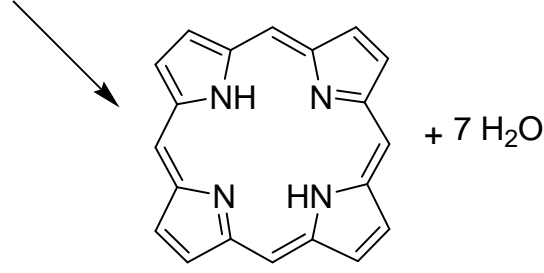

1

Scheme 1. Synthesis of porphine 1. 

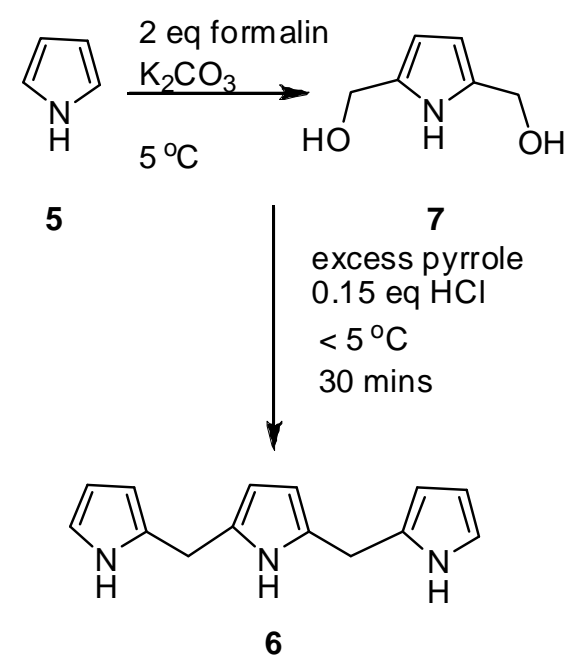

Scheme 2. Synthesis of tripyrrane 6.

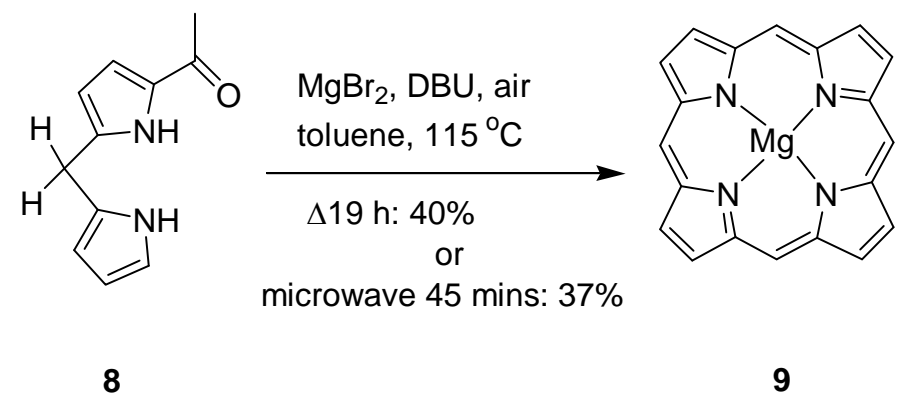

Scheme 3. Direct synthesis of (porphyrinato)magnesium(II) 9 via 1-formyldipyrromethane 8. 

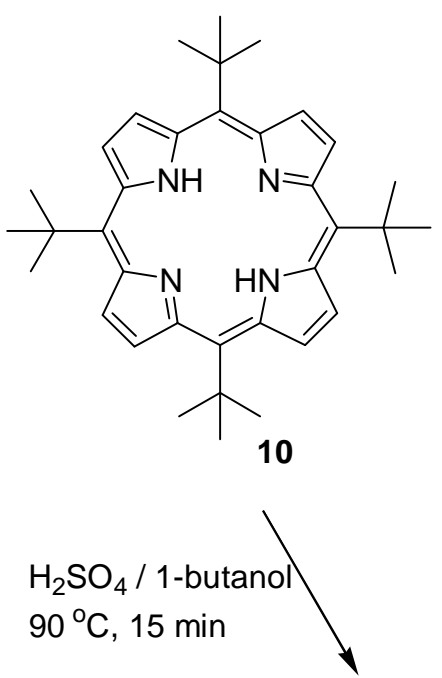

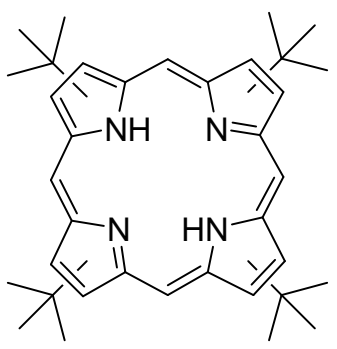

11

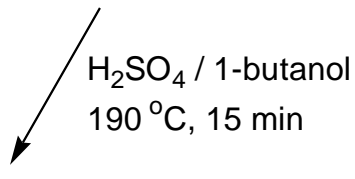

1
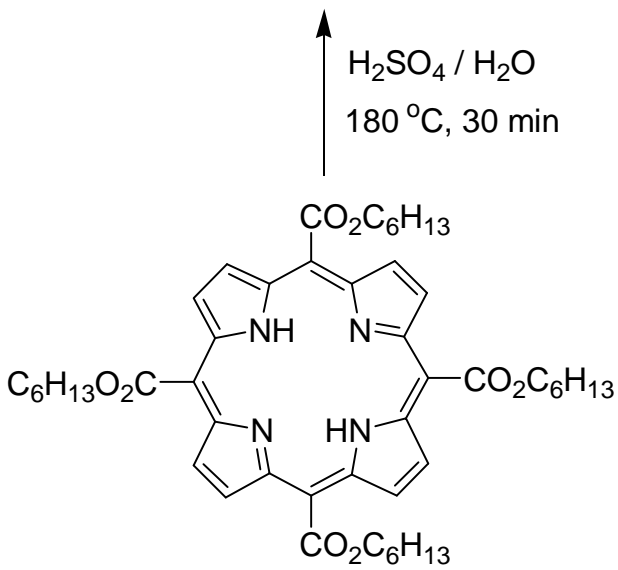

12

Scheme 4. Synthesis of porphyrin 1 through dealkylation reactions. 


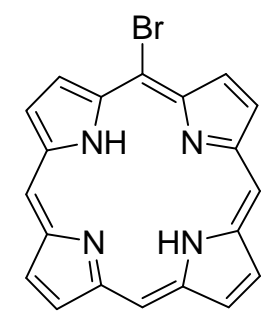

13

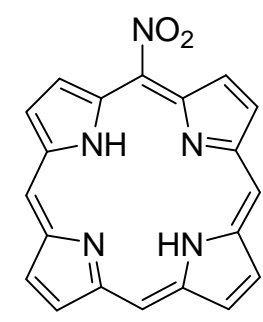

15

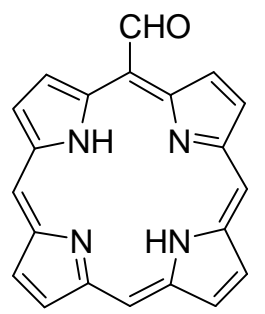

17

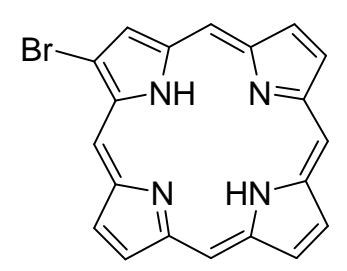

14

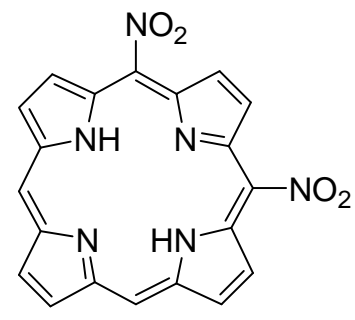

16

Formula 2 


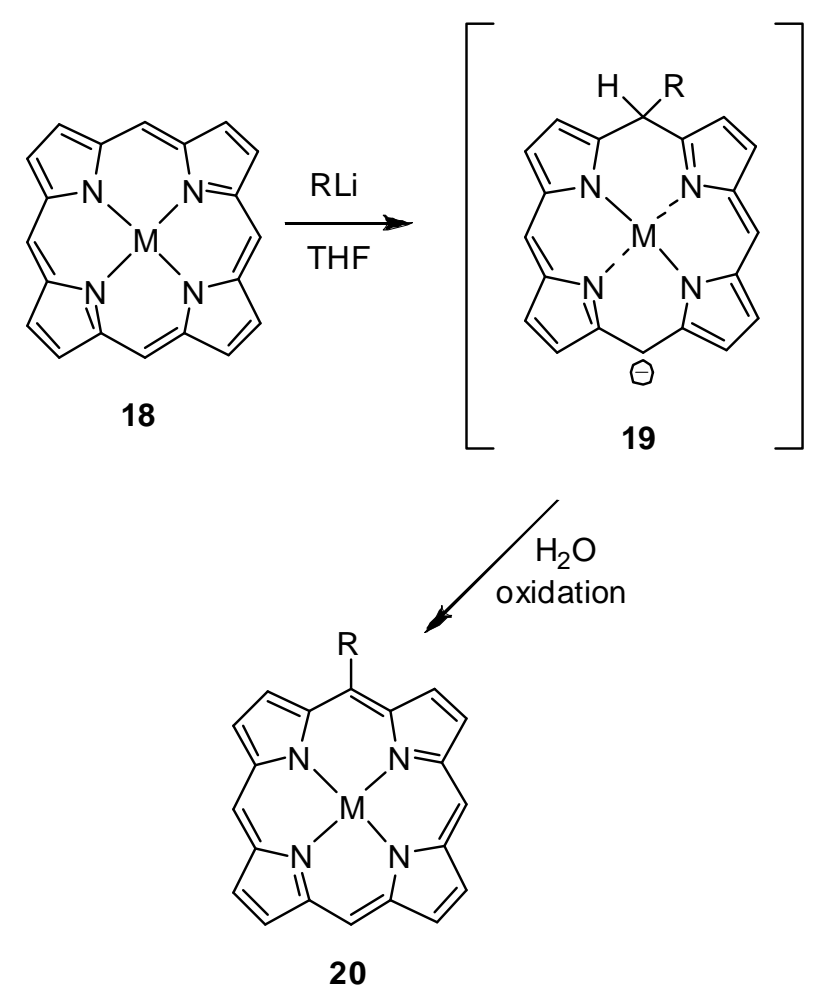

Scheme 5. meso Functionalization of porphyrin with organolithium reagents. 


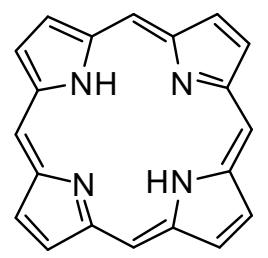

1

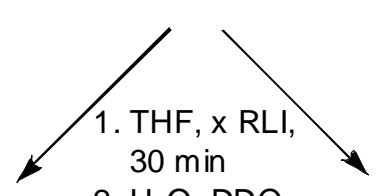

2. $\mathrm{H}_{2} \mathrm{O}, \mathrm{DDQ}$
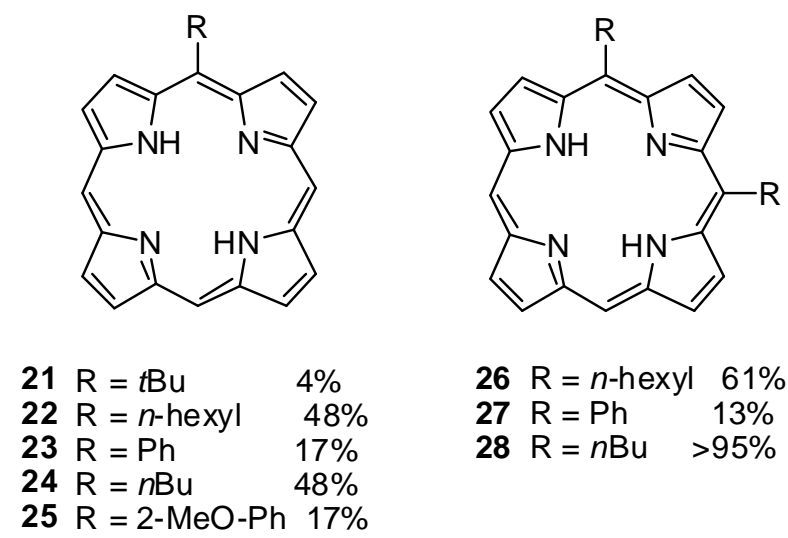

$26 \mathrm{R}=n$-hexyl $61 \%$

$27 \mathrm{R}=\mathrm{Ph} \quad 13 \%$

$28 \mathrm{R}=n \mathrm{Bu} \quad>95 \%$

$25 \mathrm{R}=2-\mathrm{MeO}-\mathrm{Ph} 17 \%$

Scheme 6. Nucleophilic substitution of porphine. 


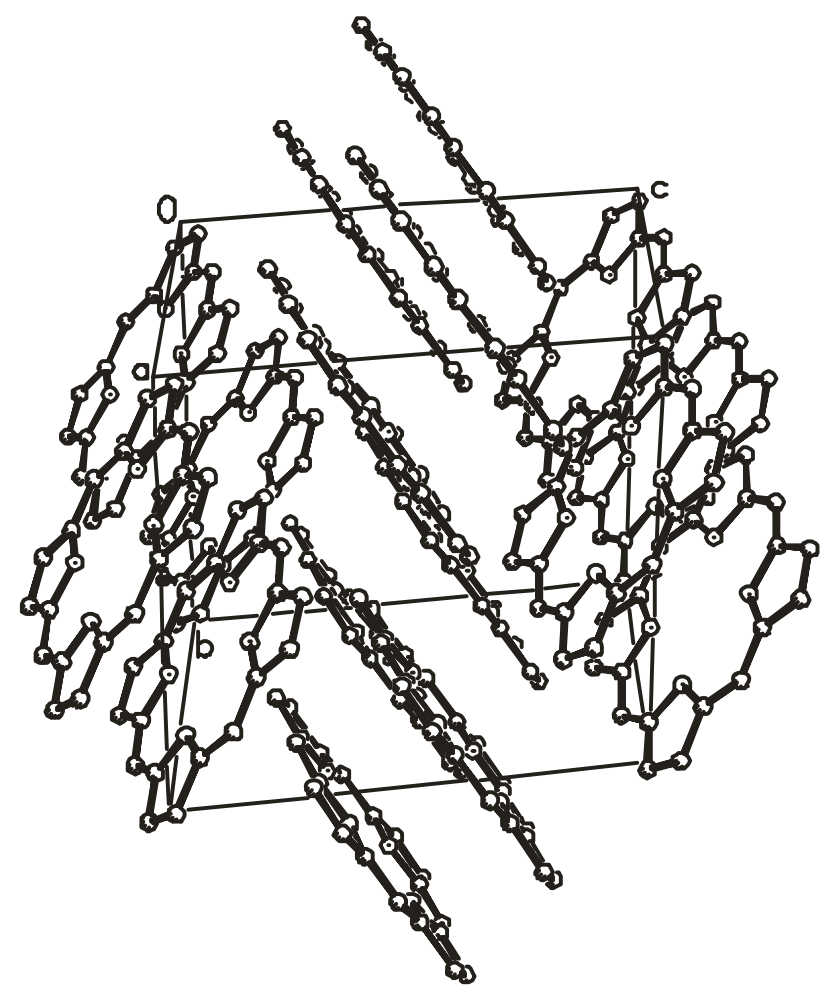

Figure 1. View of the molecular arrangement of $\mathbf{1}$ in the crystal. Hydrogen atoms have been omitted for clarity [66].
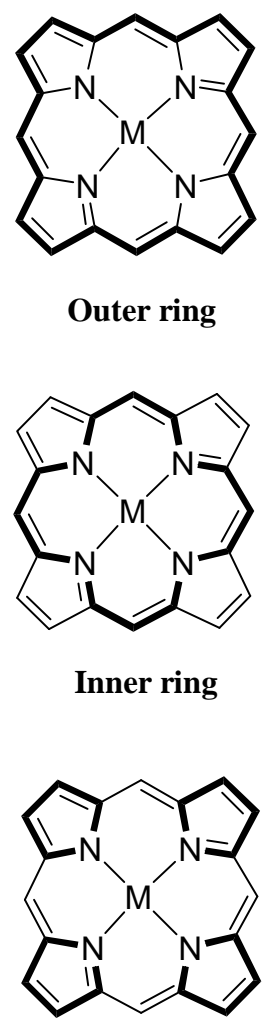

Pyrrole rings 
Figure 2: Inner ring, outer ring and pyrrole patterns [74]. 